\title{
Squamous cell carcinoma of the nail unit
}

\author{
Michela Starace ${ }^{1}$, Aurora Alessandrini ${ }^{1}$, Emi Dika $^{1}$, Bianca Maria Piraccini ${ }^{1}$
}

1 Department of Specialized, Diagnostic and Experimental Medicine, University of Bologna, Bologna, Italy

Key words: squamous cell carcinoma, malignant nail tumor, nail surgery, nail unit, onychoscopy

Citation: Starace M, Alessandrini A, Dika E, Piraccini BM. Squamous cell carcinoma of the nail unit. Dermatol Pract Concept. 2018;8(3):238244. DOI: https://doi.org/10.5826/dpc.0803a17

Received: December 31, 2017; Accepted: February 28, 2018; Published: July 31, 2018

Copyright: @2018 Starace et al. This is an open-access article distributed under the terms of the Creative Commons Attribution License, which permits unrestricted use, distribution, and reproduction in any medium, provided the original author and source are credited.

Funding: None.

Competing interests: The authors have no conflicts of interest to disclose.

All authors have contributed significantly to this publication.

Corresponding author: Michela Starace, MD, PhD, v. Massarenti 1, 40138, Bologna, Italy. Email: michela.starace2@unibo.it

ABSTRACT Squamous cell carcinoma (SCC) of the nail apparatus is a rare malignant tumor that usually originates underneath the nail plate and grows slowly with possible bone invasion. The etiology remains unknown, although a strong association with different conditions, such as high-risk human papillomavirus (HPVs), trauma, or radiation exposure has been demonstrated. Nail SCC is called "the great mimicker nail tumor" because different clinical presentations may coexist, resembling benign or malignant nail lesions. For this reason, there is often a significant delay between the onset of nail SCC and the diagnosis. Clinical manifestation includes onycholysis and erythema, while in the advanced stages nail ulceration can be observed. The association with pain, swelling, and inflammation usually indicates an invasive SCC with involvement of the underlying bone. Metastasis is rare but possible with involvement of lymph nodes. A multidisciplinary approach to assessment, management, and follow-up is advised. Using careful examination and modern diagnostic methods, including onychoscopy, biopsy, and histopathology, will help identify SCC and prevent the invasive progression. X-ray is important to investigate the bone invasion to determine the best surgical approach that will have satisfying cosmetic and functional outcomes. Nevertheless, local excision with sufficient surgical margins, best if using Mohs surgery, is usually sufficient and superior to amputation of the distal phalanx. This review aims to highlight the correct approach in suspected SCC of the nail unit.

\section{Introduction}

Squamous cell carcinoma (SCC), together with Bowen's disease, the in situ form of SCC, is the most common malignant tumor of the nail and is usually slow growing [1]. However, both have a very low incidence [2]. Several factors have been proven to favor its development, such as exposure to ionizing radiation, high-risk HPV, and chronic trauma. SCC tends to occur most commonly on the fingernails of elderly men and mainly in the thumb.
SCC has an indolent course and causes very mild symptoms. Clinical manifestations depend on the site within the nail unit where it arises (most commonly from the nail bed), and they include lateral detachment (onycholysis) of the nail and a warty aspect, with a longitudinal band of melanonychia, erosion of the nail bed associated or not with a nodule, and, rarely, longitudinal erythronychia.

There is often a substantial delay in the diagnosis of SCC in the nail unit because is often misdiagnosed as chronic paronychia, onychomycosis, pyogenic granuloma, subungual 
warts, subungual exostosis, keratoacanthoma, or amelanotic melanoma [3]. Moreover, the majority of SCCs of the nail unit originate from advanced Bowen's disease lesions, and it is therefore not surprising that lesions may be present for years, sometimes more than a decade [4].

Onychoscopy can be useful for the diagnosis, showing onycholysis, irregular vascularity, or hemorrhages with a rough-to-verrucous surface, but the features of this tumor are not exclusive; histology is mandatory. Surgical excision remains the mainstay treatment of SCC: classical surgical removal is recommended for invasive SCC, whereas Mohs micrographic surgery is indicated for noninvasive SCC. Therefore, the diagnosis of the SCC can be challenging and is often delayed, jeopardizing the possible use of a preserving surgical approach. Bone invasion and metastases are, however, rare.

\section{Epidemiology}

The prevalence of SCC ranges from $0.0012 \%$ of hospital patients to $0.028 \%$ of dermatology outpatients [2]. The typical patient is a middle-aged man with an ulcerated nodule of the nail bed or lateral onycholysis that has not been cured by previous treatments. The peak incidence age is between 50 and 69 years, but the tumor can occur at any age during adulthood. The ratio of sex incidence for male to female is $2: 1$ [5]. SCC usually involves one fingernail, especially the thumb (44\% of cases), with the second and third fingers of the dominant hand and the big toenail being other possible locations [6]. Only $16 \%$ of SCCs are located on the toenails [1].

\section{Pathogenesis}

Trauma, chronic sun or arsenic exposure, radiation, burning, genodermatoses, tobacco or immunosuppression, and HPV infection are considered to risk factors for the development of SCC [7]. Immune suppression has an important role in the development of SCC: immunocompromised patients present with the tumor at a younger age and with a shorter history than those patients with normal immune function [7]. There is increasing evidence of the role of HPV in the pathogenesis of SCC of the nail unit, as several types of HPV, especially type 16 detected on the nail unit as the only subtype, have been detected in several cases [8]. A possible method of transmission is from the genital area. The occurrence of multiple HPV-positive SCC in several nails in immunosuppressed patients further underlines the importance of HPV. Aggressive and extensive treatment and close follow-up are necessary in HPV-associated SCC, due to its higher recurrence rate, possibility of metastasis, and proliferative activity compared to HPV-negative SCC [9].
Although chronic inflammation and infections have been suggested as etiological factors in SCC, it is still unclear whether these alterations could be trigger factors for a malignant transformation or if such association is just an occasional finding [10].

\section{Clinical Manifestations}

SCC runs an indolent course and causes very mild symptoms. The clinical history describes a long-standing lesion, as tumor growth is very slow. SCC has a variety of clinical appearances that depend on the site within the nail unit where it arises, most commonly from the subungual region (nail bed) $(57.4 \%)$, rarely from the proximal or lateral nail folds $(31.5 \%)$, and exceptionally from the hyponychium (finger pulp skin) [5]. Possible signs of presentation of subungual SCC follow.

1. Lateral detachment (onycholysis) of the nail and warty aspect of the exposed nail bed and lateral nail fold that tends to ulcerate associated with an adjacent thin band of longitudinal melanonychia, which result from melanocytic activation by the tumor (Figure 1). This is typical of Bowen's disease, the hyperkeratoric variant of in situ SCC. The warty aspect of the lesion explains why it is frequently misdiagnosed as viral wart (Figure 2).

2. Painless erosion of the nail bed, appearing as a distal area of onycholysis with yellow discoloration and oozing from under the nail. The erosion is not visible unless the nail plate is cut away (Figure 3).

3. In long-standing lesions, the clinical aspect becomes that of a large nail bed erosion associated or not with a nodule (Figure 4).

4. Rarely (3\% of the cases), SCC can appear as a band of longitudinal erythronychia. All reported cases of this clinical presentation were in situ SCC, clinically indistinguishable from the more common erythronychia due to onychopapilloma (Figure 5) [11].

5. SCC involving multiple digits is an exceptional presentation, also known as synchronous SCC [12].

6. Most commonly localized hyperkeratosis could be the first appearance of SCC, partial or extensive. In this type, the nail bed is thickened and the nail plate is raised from the nail bed, inducing an onycholysis due to an accumulation of parakeratotic material [13].

Compared with SCC of the rest of the skin, nail SCC tends to become invasive more quickly. Bone involvement is seen in more than $20 \%$ of cases, ranging from $16 \%$ to $66 \%$ $[6,13,14]$, with the frequency peaks increasing in immunocompromised patients, such as AIDS patients under highly active antiretroviral therapy $[6,15]$. Pain may occur when bone invasion is present [4]. Lymph nodal involvement is 


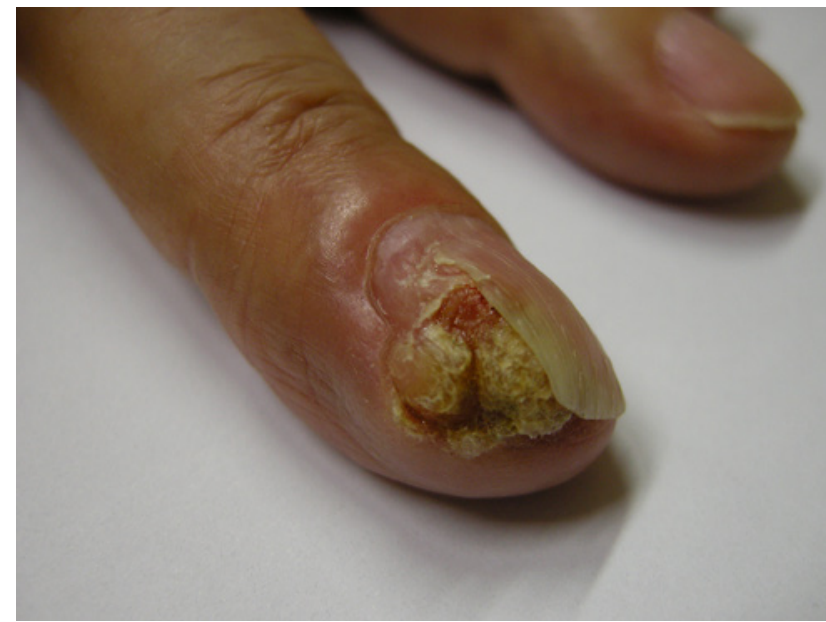

Figure 1. SCC with lateral detachment of the nail and a warty aspect of the exposed nail bed and lateral nail fold with black discoloration that tends to ulcerate with a scab formation. [Copyright: (C)2018 Starace et al.]

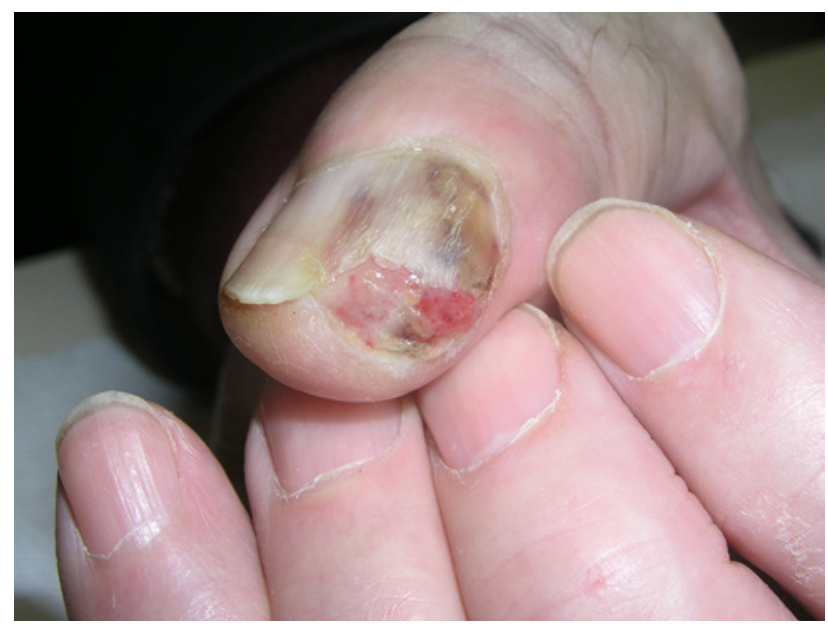

Figure 3. SCC with eroded mass under the nail, characterized by onycholytic area with oozing from the nail in late stage due to subungual erosion. [Copyright: (2018 Starace et al.]

less common, occurring in $2 \%$ of patients [16]. Tendency to metastasize is low but has been reported in a few cases [17].

\section{Differential Diagnosis}

SCC is a rare neoplasm that often mimics other entities resembling benign or common infectious or inflammatory processes of the nail apparatus. The lack of awareness among physicians, its painless history, and the higher frequency of benign diseases than malignant conditions that simulate SCC are responsible for the delay in its diagnosis [13]. Differential diagnosis includes pyogenic granuloma [18], onychomycosis [10], onychopapilloma [11], longitudinal melanonychia [19], subungual exostosis, chronic paronychia, fibrokeratoma, and onychomatricoma [1].

The most important diagnosis to rule out in case of suspect SCC is viral warts. HPV-induced warts appear as

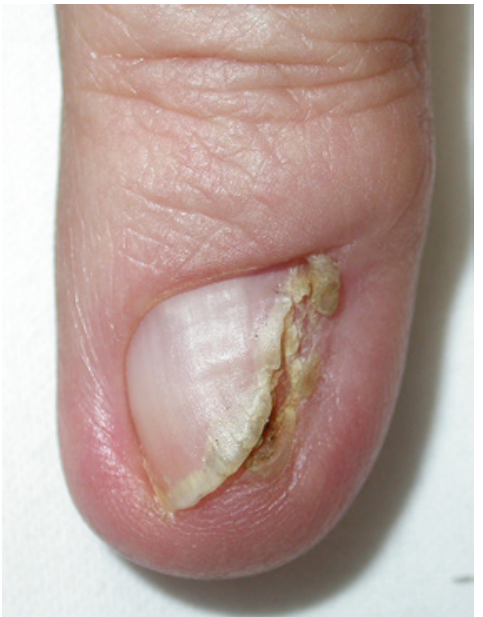

Figure 2. SCC with lateral verrucous lesion, frequently misdiagnosed as viral wart especially when the lesion is characterized by nail plate partial or total absence due to a hyperkeratotic nodule. [Copyright: @2018 Starace et al.]

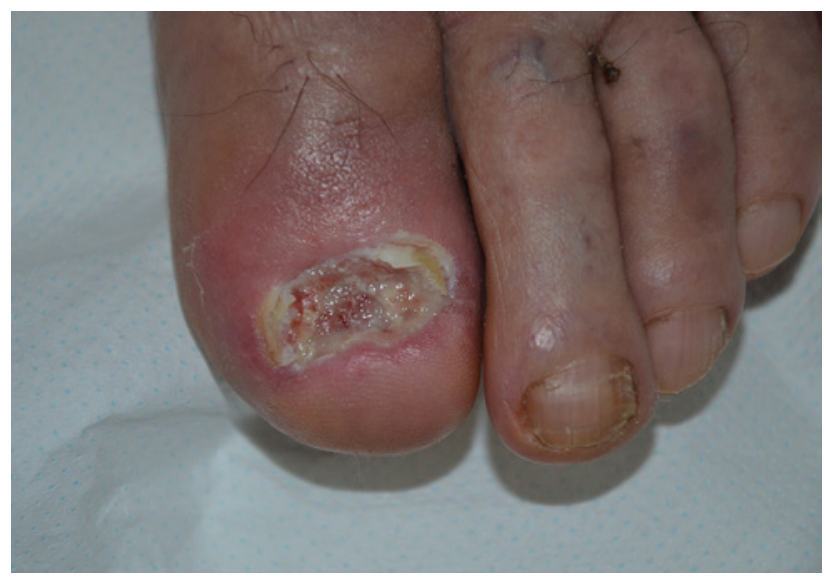

Figure 4. SCC long-standing subungual lesion, evolving in nail bed large erosion with disappearing of nail plate. [Copyright: (C2018 Starace et al.]

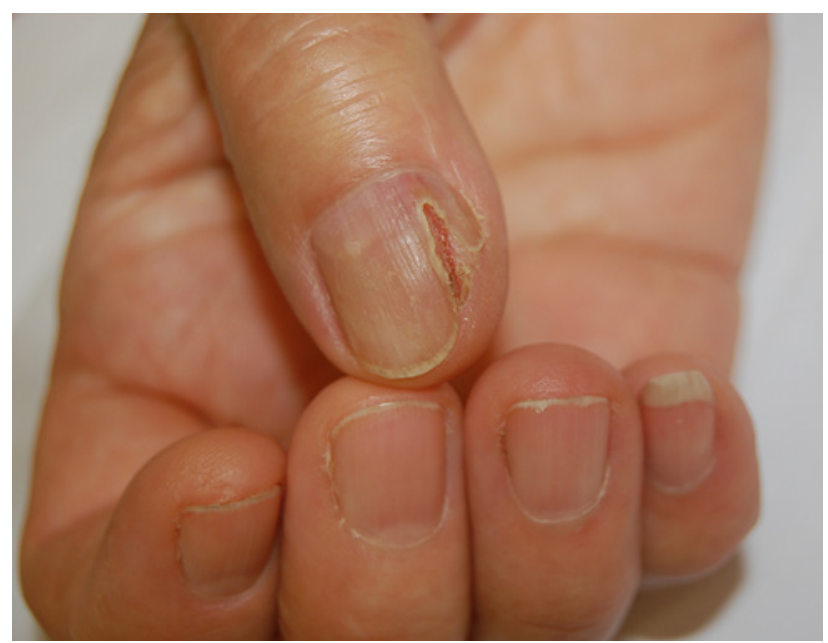

Figure 5. SCC with a longitudinal erythronychia and erosion of under nail bed. [Copyright: $@ 2018$ Starace et al.] 

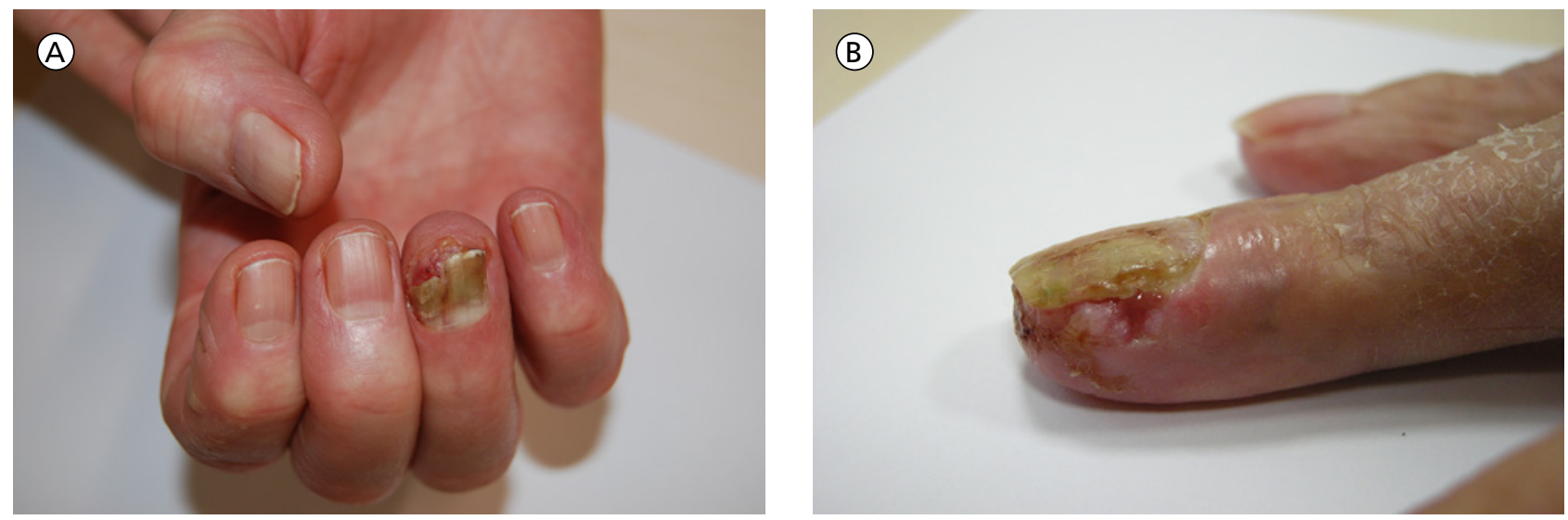

Figure 6. Clinical picture in (A) frontal view and (B) lateral view of SCC and onychoscopy of typical alterations of SCC: onycholysis, irregular vascularity, and erosion of nail bed (C) before and (D) after nail clipping onycholytic nail plate. [Copyright: @2018 Starace et al.]
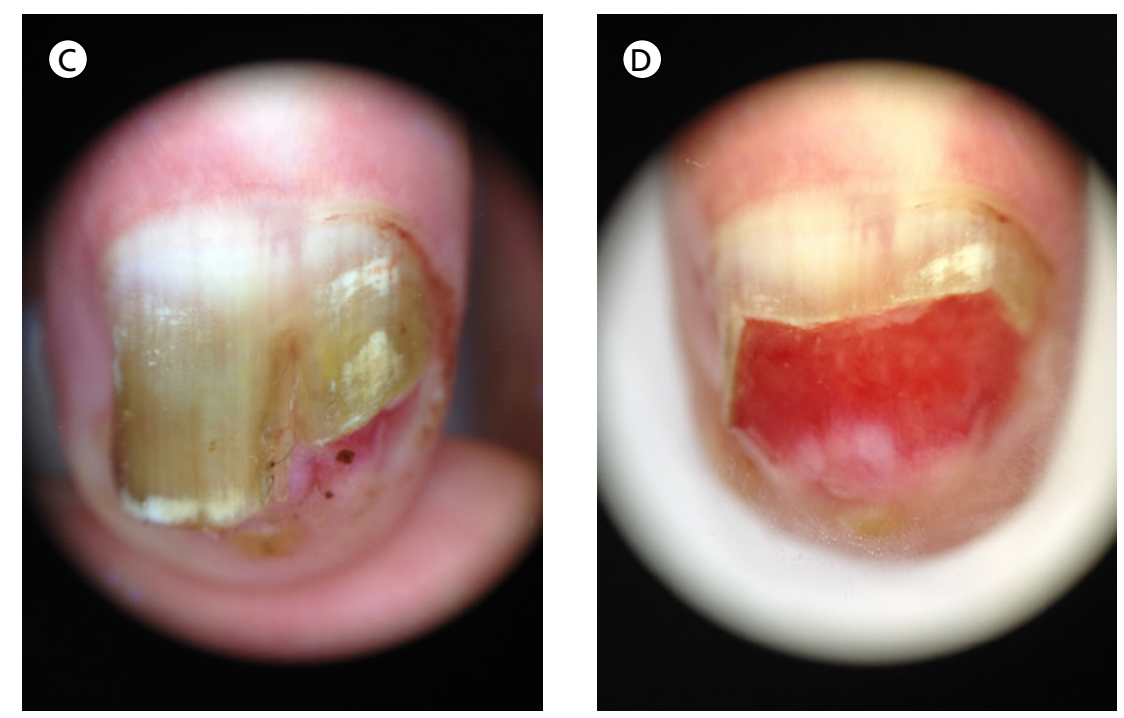

multiple hyperkeratotic and exophytic papules or nodules, especially located on the palmar or dorsal side of the finger, but may also involve the periungual skin [20]. Differential diagnosis with Bowen's disease is difficult in these cases. Another important differential diagnosis is between SCC presenting as eroded lesion of the nail bed and amelanotic melanoma [21,22].

\section{Diagnostic Techniques}

The use of nail dermoscopy (onychoscopy), a noninvasive method to better observe the nail unit, has been described as a useful instrument as a preoperative method to evaluate nail diseases for a more accurate examination than the naked eye. Under these circumstances, onychoscopy has been shown to decrease the number of unnecessary excisions of benign lesions. Typical alterations visible in SCC with onychoscopy are onycholysis, irregular vascularity, or hemorrhages with a rough to verrucous surface (Figures 6 and 7). None of these signs is, however, exclusive or diagnostic for the tumor. Differential diagnosis between periungual warts and SCC is not helped by onychoscopy as well, as the 2 types of lesions share several signs, such as yellowish and rough to verrucous

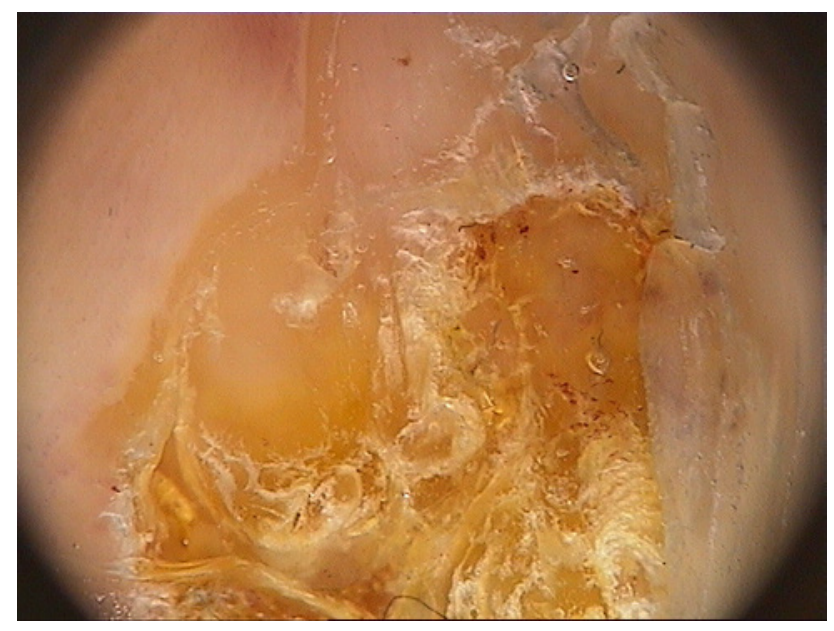

Figure 7. Onychoscopy of a verruca-like aspect of SCC: onycholysis, irregular vascularity, and hemorrhage, verrucous surface of nail bed. [Copyright: (02018 Starace et al.]

surface with brown-red streaky hemorrhages [23]. The use of onychoscopy, however, reduces the difficulties for differential diagnosis versus onychopapilloma [24], onychomatricoma [25], and subungual exostosis [26].

The vascular polymorphism is the most important aspect seen by onychoscopy in SCC producing eroded lesions of the 
nail bed and is characterized by dot-like to glomerular vessels clustering in groups [23]. In a recent paper, Teysseire et al [27] described the clinical and dermoscopic presentations of SCC finding irregular and unparalleled borders, hyperkeratosis underneath the nail plate, splinter hemorrhages, white longitudinal lines, nail thickening, and a polycyclic/fuzzy lesion edge. Some presentations are not specific to SCC, but are also observed in other diseases such as onychomatricoma or onychopapilloma, especially in the case of localized hyperkeratosis, parallel edges, sharp demarcation, and splinter hemorrhages. The onychoscopy criterion significantly associated with series of SCC of this study is unparalleled lateral edges or fuzzy edges of the nail lesion.

A method recently used to investigate nail tumors is ex vivo fluorescence confocal microscopy (FCM). Debarbieux et al [28] described the excellent correlation of malignant epithelial tumors with the observation of marked cytological and architectural atypia, such as nuclear pleomorphism and densely packed and irregularly organized nuclei, typical of invasive SCC. The while the correlation was less favorable for minimally invasive SCC with slight cytological atypia and the "blurred cell pattern." It is also suggested that this technique be used intraoperatively to make or confirm a diagnosis and to assess the surgical margins. It is defined as an alternative to classic Mohs surgery.

At ultrasonography, SCC appears as a heterogeneously hypoechoic focal mass with irregular margins; in color Doppler mode, low-resistance pulsatile flow signals within the tumor or at the periphery is evident [27].

Radiological exam should be done to evaluate underlying bone involvement, which is an indicator for amputation, but is fortunately reported in less than $20 \%$ of patients [ 29 30]. More often, x-ray of the digit may show osteolysis due to compression and not to true bone invasion. X-ray and computed tomography (CT) are useful in showing SCC as a crescent-shaped soft tissue mass with osteolytic defect of the associated phalanx, without periosteal reaction. A heterogeneous hypoechoic mass with irregular contours and posterior acoustic enhancement best represents the SCC [2].

An MRI is performed to investigate soft tissues or bone masses in the subungual area. An MRI is superior to the other radiologic imaging methods for soft tissue masses, as it has the capability of identifying the exact location and extension and adds value in local staging for SCC [2].

Accurate diagnosis can only be made by performing an appropriate biopsy followed by histopathologic evaluation [27], but biopsy is painful and often leaves definitive dystrophic scars. Therefore, many dermatologists are reluctant to perform biopsies and instead let the SCC grow, treating it as a benign lesion. As a result, the patients are often misdiagnosed, leading to an average delay of 4 years for definitive treatment, as noted in the literature [13]. Nail biopsy should, in particular, be performed in any case of a recurrent and persistent periungual warty lesion in order to make an early diagnosis of SCC and avoid extensive surgery and to preserve maximal function of the digit.

\section{Histopathology}

A variable degree of irregular and incomplete keratinization and superficial ulceration is present. A high degree of atypia with large and hyperchromatic nuclei of the basal and spinous cells, single cell necrosis, and pathological mitoses are observed with frequent keratin pearls. The epidermis shows vacuolated superficial keratinocytes with pyknotic raisin-like nuclei. In the late stage, the deep invasion of the bone can observed, and this type of SCC is negative for HPV DNA $[4,30]$.

One of the most pertinent differential diagnoses of SCC of the nail is subungual keratoacanthoma (KA). The main difference is that SCC has a very irregular stratification, while keratoacanthoma has a normal maturation of the epithelial cells from the basal layer to the center. Moreover, is possible to observe a lip formation in KA, but not in SCC, as well as the presence of elastic fibers in KA, a sign of its rapid growth, which is not seen in SCC [4].

\section{Treatment}

A variety of treatment options is available for SCC, including surgical excision, microscopically controlled surgery (Mohs surgery), therapeutic radiation, and nonsurgical alternatives. Surgical excision remains the mainstay treatment of SCC: classically, surgical removal is mandatory for invasive SCC, whereas Mohs micrographic surgery is indicated for noninvasive SCC, as it allows excision of the diseased part of the nail with minimal residual scarring, reduces the number of unnecessary amputations, and preserves the quality of life of the patient [31]. Mohs surgery also allows the evaluation of periosteal invasion to be distinguished from inflammation or compression [31]. Wide surgical excision with no less than $4 \mathrm{~mm}$ of normal tissue from the margin of the tumor can be used for the lesions without bone involvement. The postsurgical reconstruction can be done with full thickness skin graft reconstruction. A recent paper described postoperative complications of Mohs surgery: in the early phase, Topin-Ruiz et al observed graft infection and delayed wound healing with severe pain; late-stage complications included hypersensitivity to mechanical shock, mildly increased sensitivity to cold, loss of fine touch sensation, and epidermal inclusion cysts [32]. These sensitive alterations are compared to the "phantom limb pain" referred to after disarticulation and volar flap reconstructions [33]. 
Given the rarity of SCC, treatment guidelines have not been established. Although surgical excision remains the mainstay treatment of SCC, a variety of nonsurgical treatment options have also been trialed with varying success. These include photodynamic therapy, $5 \%$ fluorouracil cream, or $5 \%$ imiquimod cream after curettage; they are characterized by high incidence of relapses and absence of control of histological margins of the tumors. Radiation therapy may be helpful in the case of polydactylous disease or where surgery is difficult, especially in immunocompromised patients, although the advantages must be weighed against its potential as a causative agent in these cancers [7]. Radiation therapy has been suggested as an emerging alternative treatment option to preserve integrity of the distal finger. An alternative and underutilized approach to surgical excision in difficultto-treat cases of SCC is the photon irradiation using a water bath. This technique is recommended in the case of extensive disease that can lead to unacceptable functional morbidity or cosmetic outcomes, but its use has been limited due to concerns regarding toxicity [34]. Chemotherapy is indicated only in metastatic disease.

Amputation of the distal phalanx has the highest cure rate and is indicated in case of long-standing carcinoma or bony involvement.

Recurrence is reported in only $3.5 \%$ of patients after Mohs surgery [31] and 4\% after wide surgical excision [28] compared with conventional surgery or modified surgical procedures that vary from $28.5 \%$ to $56 \%$ according to different experiences [1]. Moreover, the recurrence is higher than skin SCC. There are several explanations for this discrepancy: most nail SCC is associated with high-risk HPV, which remains in the nail and periungual tissue and may subsequently trigger oncogenic mutations. Furthermore, the surgical procedure is more difficult in nail involvement than skin areas for margins, and the pathology can be more challenging to interpret [35].

\section{Conclusions}

SCC presents with a variety of clinical appearances and course. Physicians need to maintain heightened awareness, and chronic, non-healing lesions of the digits should be viewed with suspicion. Rapidly growing ulcerative lesions should be considered as potential malignancy. The prognosis of SCC is very good if it is recognized at an early stage, highlighting the need for clipping away the nail plate and taking a biopsy in patients with chronic or recurrent oozing from under the nail that fails to respond to any previous conservative treatment. Nail dermoscopy is useful in looking for longitudinal melanonychia or erythronychia, irregular vascularity, and hemorrhages. Moreover, it is helpful to better visualize the onycholytic nail plate and subungual hyperkeratosis with high magnification that permits observation of the aspect of the color and shape. It is not a specific method for diagnosis, but it is helpful in differential diagnosis in some conditions such as onychomatricoma and onychomycosis. Another important role of dermoscopy can be the selection of the biopsy site [27]. Pathological confirmation is necessary for early diagnosis and treatment effectiveness. The presence of pain indicates bone invasion by the tumor, and x-ray is mandatory in these patients to investigate the bone involvement. Given the rarity of the condition, there is no consensus on the optimal treatment. No standardized therapeutic approach is described in SCC, and the choice is selected on the basis of the extension of the tumor and the involvement of the underlying structures. Microscopic surgery and local removal are advised in superficial lesions for the lower recurrence rate, while large excision until amputation are recommended for patients with bone infiltration. The tendency of recurrence is higher in the nail unit than in another part of the body, likely due to residual HPV in the surrounding area or an incomplete excision of the tumor, and for this reason a strict and long follow-up is recommended for SCC.

\section{References}

1. Lecerf P, Richert B, Theunis A, André J. A retrospective study of squamous cell carcinoma of the nail unit diagnosed in a Belgian general hospital over a 15 -year period. J Am Acad Dermatol. 2013;69(2):253-261. doi: 10.1016/j.jaad.2013.02.008.

2. High WA, Tyring SK, Taylor RS. Rapidly enlarging growth of the proximal nail fold. Dermatol Surg. 2003;29(9):984-986.

3. Tambe SA, Patil PD, Saple DG, Kulkarni UY. Squamous cell carcinoma of the nail bed: the great mimicker. J Cutan Aesthet Surg. 2017;10(1):59-60. doi: 10.4103/JCAS.JCAS_35_16.

4. Haneke E. Important malignant and new nail tumors. J Dtsch Dermatol Ges. 2017;15(4):367-386.

5. de Berker DA, Dahl MG, Malcolm AJ, Lawrence CM. Micrographic surgery for subungual squamous cell carcinoma. Br J Plast Surg. 1996;49(6):414-419. doi: 10.1016/S00071226(96)90013-2.

6. Kelly KJ, Kalani AD, Storrs S, et al. Subungual squamous cell carcinoma of the toe: working toward a standardized therapeutic approach. J Surg Educ. 2008;65(4):297-301. doi: 10.1016/j. jsurg.2008.05.013.

7. Ormerod E, de Berker D. Nail unit squamous cell carcinoma in people with immunosuppression. Br J Dermatol. 2015;173(3):701712. doi: 10.1111/bjd.13860.

8. Dika E, Venturoli S, Patrizi A, et al. The detection of human papillomavirus-16 in squamous cell carcinoma of the nail unit: A case series. J Am Acad Dermatol. 2017;76(2):354-356. doi: 10.1016/j. jaad.2016.08.063.

9. Riddel C, Rashid R, Thomas V. Ungual and periungual human papillomavirus-associated squamous cell carcinoma: a review. J Am Acad Dermatol. 2011;64(6):1147-1153. doi: 10.1016/j. jaad.2010.02.057. 
10. Grigorov Y, Philipov S, Patterson J, et al. Subungual squamous cell carcinoma associated with long standing onychomycosis: aggressive surgical approach with a favourable outcome. Open Access Maced J Med Sci. 2017;5(4):480-482. doi: 10.3889/ oamjms.2017.116.

11. Jellinek NJ, Lipner SR. Longitudinal Erythronychia: Retrospective Single-Center Study Evaluating Differential Diagnosis and the Likelihood of Malignancy. Dermatol Surg. 2016;42(3):310-319. doi: 10.1097/DSS.0000000000000594.

12. Abner S, Redstone J, Chowdhry S, Kasdan ML, Wilhelmi BJ. Synchronous squamous cell carcinoma in multiple digits. Eplasty. 2011;11:e9.

13. Meesiri S. Subungual squamous cell carcinoma masquerading as chronic common infection. J Med Assoc Thai. 2010;93(2):248251.

14. Dalle S, Depape L, Phan A, Balme B, Ronger-Savle S, Thomas L. Squamous cell carcinoma of the nail apparatus: clinicopathological study of 35 cases. Br J Dermatol. 2007;156(5):871-874. doi: 10.1111/j.1365-2133.2006.07744.x.

15. Handisurya A, Rieger A, Bankier A, et al. Human papillomavirus type 26 infection causing multiple invasive squamous cell carcinomas of the fingernails in an AIDS patient under highly active antiretroviral therapy. Br J Dermatol. 2007;157(4):788-794. doi: 10.1111/j.1365-2133.2007.08094.x.

16. Zaiac MN, Weiss E. Mohs micrographic surgery of the nail unit and squamous cell carcinoma. Dermatol Surg. 2001;27(3):246251.

17. Batalla A, Feal C, Rosón E, Posada C. Subungual squamous cell carcinoma: a case series. Indian J Dermatol. 2014;59(4):352-354. doi: 10.4103/0019-5154.135480.

18. Khullar G, Singh S, Saikia UN, Kumar A, Singh MP, Kanwar AJ. Squamous cell carcinoma of the nail fold masquerading as pyogenic granuloma. Indian J Dermatol Venereol Leprol. 2016;82(5):555-557. doi: 10.4103/0378-6323.182975.

19. Gatica-Torres M, Arguello-Guerra L, Manuel Ruiz-Matta J, Dominguez-Cherit J. Subungual pigmented squamous cell carcinoma presenting as a grey longitudinal melanonychia in a young patient. BMJ Case Rep. 2016;2016:2016.

20. Inkaya E, Sayit E, Sayit AT, Zan E, Bakirtas M. Subungual squamous cell carcinoma of the third finger with radiologic and histopathologic finding: a report case. J Hand Microsurg. 2015;7(1):194-198. doi: 10.1007/s12593-014-0152-4.

21. Richert B, Lecerf P, Caucanas M, André J. Nail tumors. Clin Dermatol. 2013;31(5):602-617. doi: 10.1016/j.clindermatol. 2013.06.014.

22. Starace M, Dika E, Fanti PA, et al. Nail apparatus melanoma: dermoscopic and histopathologic correlations on a series of 23 patients from a single centre. J Eur Acad Dermatol Venereol. 2018;32(1):164-173.
23. Haenssle HA, Blum A, Hofmann-Wellenhof R, et al. When all you have is a dermatoscope-start looking at the nails. Dermatol Pract Concept. 2014;4(4):11-20.

24. Tosti A, Schneider SL, Ramirez-Quizon MN, Zaiac M, Miteva M. Clinical, dermoscopic, and pathologic features of onychopapilloma: A review of 47 cases. J Am Acad Dermatol. 2016;74(3):521526. doi: 10.1016/j.jaad.2015.08.053.

25. Lesort C, Debarbieux S, Duru G, Dalle S, Poulhalon N, Thomas L. Dermoscopic features of onychomatricoma: a study of 34 cases. Dermatology. 2015;231(2):177-183. doi: 10.1159/000431315.

26. Piccolo V, Argenziano G, Alessandrini AM, Russo T, Starace M, Piraccini BM. Dermoscopy of subungual exostosis: a retrospective study of 10 patients. Dermatology. 2017;233(1):80-85. doi: 10.1159/000471800.

27. Teysseire S, Dalle S, Duru G, et al. Dermoscopic Features of Subungual Squamous Cell Carcinoma: A Study of 44 Cases. Dermatology. 2017;233(2-3):184-191. doi: 10.1159/000479059.

28. Debarbieux S, Gaspar R, Depaepe L, Dalle S, Balme B, Thomas L. Intraoperative diagnosis of nonpigmented nail tumours with ex vivo fluorescence confocal microscopy: 10 cases. $\mathrm{Br}$ J Dermatol. 2015;172(4):1037-1044. doi: 10.1111/bjd.13384.

29. Peterson SR, Layton EG, Joseph AK. Squamous cell carcinoma of the nail unit with evidence of bony involvement: a multidisciplinary approach to resection and reconstruction. Dermatol Surg. 2004;30(2 Pt 1):218-221.

30. Zavos G, Karidis NP, Tsourouflis G, et al. Nonmelanoma skin cancer after renal transplantation: a single-center experience in 1736 transplantations. Int J Dermatol. 2011;50(12):14961500. doi: 10.1111/j.1365-4632.2011.04939.x.

31. Dika E, Fanti PA, Patrizi A, Misciali C, Vaccari S, Piraccini BM. Mohs Surgery for Squamous Cell Carcinoma of the Nail Unit: 10 Years of Experience. Dermatol Surg. 2015;41(9):1015-1019.

32. Topin-Ruiz S, Surinach C, Dalle S, Duru G, Balme B, Thomas L. Surgical treatment of subungual squamous cell carcinoma by wide excision of the nail unit and skin graft reconstruction: an evaluation of treatment efficiency and outcomes. JAMA Dermatol. 2017;153(5):442-448. doi: 10.1001/jamadermatol.2017.0014.

33. Björkman A, Weibull A, Olsrud J, Ehrsson HH, Rosén B, Björkman-Burtscher IM. Phantom digit somatotopy: a functional magnetic resonance imaging study in forearm amputees. Eur J Neurosci. 2012;36(1):2098-2106. doi: 10.1111/j.14609568.2012.08099.x.

34. Goodman CR, DeNittis A. Photon irradiation using a water bath technique for treatment of confluent carcinoma in situ of the hand, digits, and nail bed: a case report. J Med Case Reports. 2017;11(1):86. doi: 10.1186/s13256-017-1233-3.

35. Tang N, Maloney ME, Clark AH, Jellinek NJ. A retrospective study of nail squamous cell carcinoma at 2 institutions. Dermatol Surg. 2016;42(suppl 1):S8-S17. doi: 10.1097/DSS.0000000000000521. 